\title{
RDA, el nuevo código de catalogación: cambios y desafíos para su aplicación
}

\author{
Paola Picco* y Virginia Ortiz Repiso**
}

Resumen: Se estudia el nuevo código de catalogación Descripción y Acceso a los Recursos (RDA) y su adecuación a la situación tecnológica de la actualidad. Se analiza la forma en que se originó, qué representa y qué puede significar para la catalogación y para el desarrollo de los catálogos. Se hace un breve recorrido por la situación del control bibliográfico y su relación con la tecnología para continuar con los antecedentes que le dan origen al nuevo código. Asimismo se estudian sus características, los cambios que propone y qué beneficios aporta el hecho de estar basado en el modelo FRBR. Se muestran sus implementaciones en la comunidad internacional. Y, por último, se destacan los aciertos y los desafíos que supone y las dudas más relevantes que suscita. Se realiza una amplia revisión bibliográfica que ha permitido identificar los aspectos más importantes de este cambio de normalización catalográfica así como sus aciertos e inconvenientes. Asimismo se lleva a cabo un análisis minucioso de los Requisito Funcionales para Registros Bibliográficos (FRBR), de los Requisitos Funcionales para los Datos de Autoridad (FRAD) y de las instrucciones del nuevo código los que ha permitido detectar las conexiones que existen entre ellos.

Palabras clave: catalogación, FRBR, RDA, estándares catalográficos, linked data, cloud computing.

\section{$R D A$, the new cataloging code: changes and challenges}

Abstract: An in-depth study presenting the new cataloging code, RDA (Resource Description and Access) and its relationship to technology. Its origin, what it represents and its implications for cataloging are analyzed. The state of bibliographic control and the foundations of the new cataloging code are reviewed. Details are presented concerning its structure, changes and advantages of being based on the FRBR model. Reference is also made to RDA's adoption by the international community. Finally, the article sets forth the changes and doubts that RDA prompts. The exhaustive bibliographic review permits the identification of the most significant changes as well as the pros and cons. Likewise, a detailed study of the Functional Requirements of Bibliographic Records (FRBR) and the Functional Requirements for Autbority Data (FRAD) enables a better understanding of the new code's instructions, connections and relationships to them.

Keywords: cataloging, FRBR, RDA, cataloging standards, linked data, cloud computing.

* Escuela Universitaria de Bibliotecología y Ciencias Afines, Universidad de la República. Uruguay. Correo-e: picco.paola@gmail.com.

** Departamento de Biblioteconomía y Documentación, Universidad Carlos III de Madrid. Correo-e: virginia@bib.uc3m.es.

Recibido: 29-03-2011; 2. ${ }^{a}$ versión: 24-07-2011; aceptado: 29-07-2011. 


\section{Introducción}

Las bibliotecas, desde la década de los noventa, son conscientes de que la información que deben ofrecer al usuario se encuentra no solo en su propia casa sino también, en la de otros. El universo de información que, hoy en día, deben manejar, organizar y gestionar es amplio y muy diverso. También lo es la tecnología que tienen que utilizar. No solo es suficiente implantar un Sistema Integrado de Gestión de Bibliotecas, sino que es necesario dar acceso a múltiples y variados documentos que proceden de fuentes diversas y que, a veces, traen consigo una tecnología propia.

Las revistas electrónicas, los repositorios institucionales, los documentos en la red, las redes sociales, los libros electrónicos, los libros tradicionales en papel, las imágenes, los sonidos y un etcétera que se amplía y amplia llegando a la nube (Cloud computing) han propiciado nuevas herramientas de búsqueda y acceso a la información: servidores de enlaces y metabuscadores que permiten al usuario un mejor y fácil acceso. Sin olvidarnos de los nuevos servicios que comienzan a implantarse: información directa al móvil, préstamo de lectores y de libros electrónicos...

Y, claro, los catálogos bibliotecarios existen y siguen siendo necesarios. Aunque hayan pasado de ser un elemento central a un servicio, entre otros, para acceder a la información. Y, a pesar de que deban competir, además, con servicios, comerciales y/o gratuitos, cada vez más potentes y eficaces que proyectan una sombra grande sobre ellos, siguen siendo necesarios. Ahora bien, hace ya tiempo que la comunidad bibliotecaria demanda la necesidad de nuevas herramientas para su creación, difusión y búsqueda. Y, además, la necesidad de simplificar la catalogación. Las ISBD (IFLA, 2007), las AACR (2005), las normas catalográficas nacionales y, también, el formato MARC (Library of Congress, 2009a) arrastran el peso del entorno manual en el que fueron creadas y de la tecnología que estaba disponible en los años sesenta.

Desde hace unos años, los acrónimos FRBR, FRAD, RDA se van haciendo más presentes entre nosotros. Las dudas, las inquietudes y, también, las expectativas que se han creado son muchas. La primera pregunta que nos hacemos ¿es realmente algo nuevo y acorde con los tiempos? ¿Los catálogos van a asemejarse a las herramientas que mayoritariamente se utilizan en la web? ¿Son necesarias más normas?

En el mes de julio de 2010, se publicó, después de diez años de discusiones entre la comunidad internacional de catalogadores un nuevo código de catalogación: Resource Description and Access conocido bajo el acrónimo RDA (2010) que sustituirá a las Anglo American Cataloguing Rules (AACR).

Lo que a continuación vamos a exponer es un análisis del estado de la cuestión. Supone el primer paso de una investigación, en curso, cuyo objetivo último consiste en estudiar las RDA y su adecuación a la situación tecnológica de la actualidad, proponiendo soluciones de implantación.

En este trabajo queremos estudiar cómo se origina este nuevo código, qué representa y qué puede significar. Para conseguir este objetivo haremos, en pri- 
mer lugar, un breve recorrido por la situación del control bibliográfico y su relación con la tecnología para continuar con los antecedentes que lo han originado. Analizaremos, en segundo lugar, sus características, los cambios que propone y qué beneficios aporta el hecho de estar basado en el modelo FRBR. En el tercer paso, mostraremos sus implementaciones en la comunidad internacional. Y, por último, indicaremos los aciertos y los desafíos que supone y las dudas más relevantes que suscita.

\section{Aspectos metodológicos}

Para la realización de este estudio, se ha llevado a cabo una exhaustiva revisión bibliográfica que ha permitido identificar los aspectos más importantes de este cambio de normalización catalográfica, así como sus aciertos e inconvenientes.

Se ha realizado, además, un análisis de los Requisito Funcionales para Registros Bibliográficos (IFLA, 1998) de los Requisitos Funcionales para los Datos de Autoridad (FRAD) (IFLA, 2009b), así como de las RDA (2010) que han permitido detectar las conexiones que existen entre ellos.

Se ha llevado a cabo una comparación entre el formato MARC 21, las AACR y las RDA, que nos ha permitido identificar los cambios producidos y cómo se reflejan en la elaboración de los registros bibliográficos. Hemos consultado el Plan estratégico de evaluación del nuevo código que viene realizando la Library of Congress junto a las dos Bibliotecas Nacionales norteamericanas y otras instituciones de este mismo país que forman parte del proceso de manera voluntaria (Library of Congress, 2009c). Asimismo, se realizaron consultas a catálogos que ya están aplicando este estándar. Se compararon registros bibliográficos generados a partir de la aplicación de los dos códigos, se analizaron sus semejanzas y diferencias.

Para conocer el estado en el que se encuentra la adopción del nuevo código analizamos, tanto el Plan estratégico de evaluación del Código de la Library of Congress (2009c), como los documentos y las políticas definidas por OCLC (2010), la Biblioteca Nacional de Australia (National Library of Australia, 2010) y el informe final del U.S. RDA Test Coordinating Committee (2011). Así como los documentos de las Bibliotecas Nacionales de los diferentes países de Europa (RDA in Europe, 2010; Hernández, 2010).

\section{El control bibliográfico y los estándares}

El control bibliográfico tiene como objetivo fundamental desarrollar herramientas que permitan la organización de la información para su posterior recuperación y acceso. Se sostiene sobre una serie de principios y prácticas comunes que fueron adquiriendo relevancia en el ámbito internacional desde los años 
sesenta y que han facilitado el intercambio de la información y la racionalización de los recursos a través de proyectos de cooperación y trabajo colectivo. Los Principios de Paris, las normas ISBD, el formato MARC 21, las AACR son herramientas de control bibliográfico que cumplen distintas funciones pero que se complementan para alcanzar un objetivo común. Seguramente los catalogadores estamos familiarizados con las mismas, pero, sin embargo, hemos observado que muchas veces se confunde el alcance de cada una de ellas. En este epígrafe nos proponemos demostrar cómo cada herramienta del sistema de control bibliográfico cumple una tarea específica. Pero también, como todas se complementan buscando alcanzar el objetivo general del sistema, que es organizar la información para hacerla accesible a la comunidad de usuarios.

En este mismo sentido, Sevenonius (2000) explica las funciones que cumplen los principios (Principios de Paris, Principios Internacionales de Catalogación), objetivos y lenguajes bibliográficos (reglas de catalogación) dentro del sistema de control bibliográfico. La autora sostiene que los principios son reglas generales que van a orientar el diseño de los lenguajes bibliográficos, que los objetivos definen las acciones esperadas por el sistema, teniendo en cuenta las necesidades de los usuarios, y que el lenguaje bibliográfico, toma forma de instrucciones precisas o reglas, que son las que utilizamos para describir a los distintos recursos de información.

Por otra parte, Gorman (2006) manifiesta que un sistema de control bibliográfico está compuesto por principios, estándares, reglas de descripción y aplicaciones informáticas, así como por las interpretaciones realizadas a nivel local. Como ejemplo de reglas de descripción, cita las AACR, y el sistema de Encabezamiento de Materias de la Library of Congress. Para el caso de aplicaciones informáticas, el formato MARC, y respecto a las interpretaciones, las realizadas a las AACR por la Library of Congress.

Pero ahora el espectro ha cambiado, no solo hablamos de un control bibliográfico pura y exclusivamente bibliotecario con normas internacionales propias. Nos encontramos con recursos de información que son organizados por otras instituciones según sus propias prácticas y que deben convivir con las colecciones tradicionales de las bibliotecas. De esta forma, tenemos junto a las colecciones tradicionales, que se procesan a partir de las prácticas consensuadas de la Catalogación, bases de datos bibliográficas producidas por consorcios comerciales, así como repositorios digitales desarrollados según dinámicas y reglas propias, también internacionalmente consensuadas. Pero, en todo caso, debemos subrayar la diversidad de los elementos que la biblioteca deberá gestionar para ponerlos a disposición de la comunidad de usuarios, buscando satisfacer sus necesidades de la forma más eficiente y adecuada a sus intereses.

Las herramientas del sistema de control bibliográfico están en estrecha relación con las tecnologías. La utilización de los Sistemas Integrados de Gestión de Bibliotecas permite la generación de los OPAC. Los servidores de enlace muestran las fuentes disponibles para un recurso determinando sin importar su ubicación. Los metabuscadores permiten realizar búsquedas federadas en el universo de la 
biblioteca. La tecnología disponible para las bibliotecas ha evolucionado al punto de permitir brindar servicios en la telefonía móvil, consultas a los OPAC, servicios de diseminación selectiva de información y hasta la descarga de documentos licenciados por la biblioteca.

Las herramientas de control bibliográfico y, fundamentalmente, de acceso a la información han ido evolucionando a partir de nuevos desarrollos tecnológicos y nuevos estándares conformando un todo. En la figura 1, se muestra una representación de los estándares que conforman las herramientas de control bibliográfico, comparando el esquema propuesto por W3C Library Linked Data Incubator Group (2005), al que haremos referencia más adelante, y la actualización que proponen las autoras.

\section{FIGURA 1}

\section{Estándares para el control bibliográfico}

Estándares bibliográficos definidos por W3 Library Linked Data Incubator Group, 2005

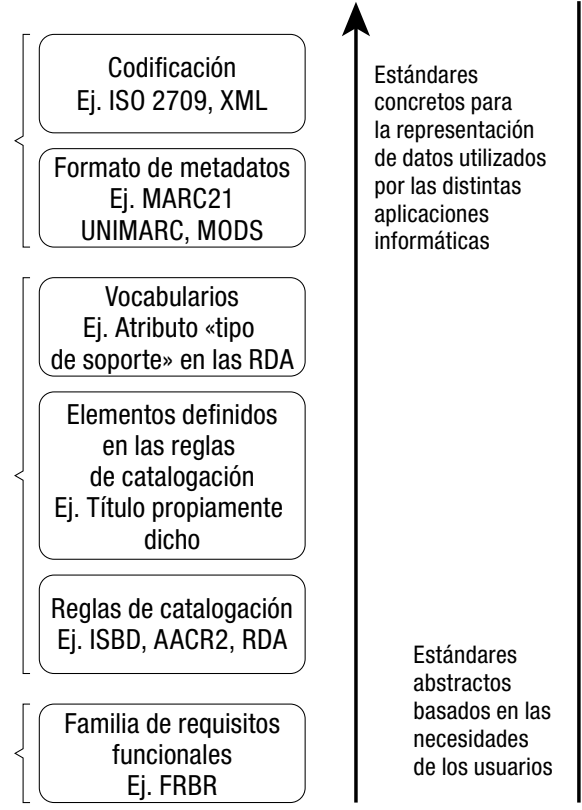

Actualización propuesta por las autoras

\begin{tabular}{|c|c|}
\hline \multicolumn{2}{|c|}{ APLICACIONES INFORMÁTICAS } \\
\hline \multirow{2}{*}{ ESTRUCTURA } & $\begin{array}{l}\text { Codificación } \\
\text { Ej. ISO 2709, XML }\end{array}$ \\
\hline & $\begin{array}{c}\text { Formato de metadatos } \\
\text { Ej. MARC21 } \\
\text { UNIMARC, MODS }\end{array}$ \\
\hline \multicolumn{2}{|c|}{ NIVEL DE REPRESENTACIÓN DE DATOS } \\
\hline \multirow{3}{*}{ CONTENIDO } & $\begin{array}{c}\text { Vocabularios } \\
\text { Ej. Atributo «tipo } \\
\text { de soporte» en las RDA }\end{array}$ \\
\hline & $\begin{array}{l}\text { Elementos definidos } \\
\text { en las reglas } \\
\text { de catalogación } \\
\text { Ej. Título propiamente } \\
\text { dicho }\end{array}$ \\
\hline & $\begin{array}{l}\text { Reglas de catalogación } \\
\text { Ej. ISBD, AACR2, RDA }\end{array}$ \\
\hline \multicolumn{2}{|c|}{ NIVEL ABSTRACTO } \\
\hline \multirow{2}{*}{$\begin{array}{l}\text { MODELOS } \\
Y \\
\text { PRINCIPIOS }\end{array}$} & $\begin{array}{l}\text { Principios de catalogación } \\
\text { Ej. Principios de París } \\
\text { Declaración internacional } \\
\text { de Principios } \\
\text { de Catalogación }\end{array}$ \\
\hline & $\begin{array}{c}\text { Familia de requisitos } \\
\text { funcionales } \\
\text { Ej. FRBR, FRAD, FRSAD }\end{array}$ \\
\hline
\end{tabular}


En nuestra propuesta agregamos, a los Principios de Catalogación, en un escalón posterior a los modelos, y señalamos tres niveles: uno abstracto que incluye a los modelos y principios, el segundo de representación de datos, donde se encuentran los vocabularios controlados, y las reglas de catalogación. Y, el tercero, con las aplicaciones informáticas, que incluye los formatos de almacenamiento y los lenguajes de codificación.

El nuevo código de catalogación está vinculado, como ya se ha señalado, a los modelos FRBR y FRAD que le dan el sustento teórico. Muchas veces se produce cierta confusión entre marco conceptual, principios y estándares. Creemos necesario hacer un pequeño paréntesis para explicar el significado de cada uno de ellos.

Los marcos conceptuales son representaciones abstractas que se utilizan para tratar de explicar un fenómeno o proceso (Carlyle, 2006). Son en general representaciones de una realidad, o simulaciones, que permiten comprender como suceden las cosas en determinada circunstancia, con todas las limitaciones que pueden tener por ser interpretaciones concebidas por la menta humana. Pero más allá de sus propias limitaciones, son de mucha utilidad para las distintas disciplinas. Los modelos FRBR y FRAD son marcos conceptuales y no reglas de catalogación, tienen como objetivo explicar el universo bibliográfico a través de la definición de una serie de entidades, sus atributos y relaciones, como veremos en el próximo epígrafe.

Los principios son guías o directrices que van a orientar la definición de los estándares de descripción. Estos últimos son instrucciones o reglas específicas que nos permiten representar de manera simbólica al universo bibliográfico. Entendemos también por estándares a los de almacenamiento que son aplicaciones informáticas que recogen de forma sistemática las descripciones y representaciones de esa realidad como es el caso del formato MARC21. Para registrar la información en ese formato se siguen las AACR, combinándose la aplicación de estos dos estándares (uno para describir, el otro para almacenar).

Una vez realizada esta puntualización y las correspondientes aclaraciones, analizaremos a continuación los antecedentes del nuevo código de catalogación, para detenernos después en el análisis de los cambios que supone su utilización a partir de la incorporación de los modelos conceptuales en sus bases teóricas.

\section{Antecedentes del código RDA}

En la última década del siglo xx, los organismos internacionales, principalmente la IFLA, comenzaron a trabajar para dotar a los catálogos de una estructura y de unas normas que estuvieran más acordes con el entorno tecnológico existente. Los estándares catalográficos vigentes están pensados para un entorno manual, sin embargo, el avance tecnológico exige una actualización de las normas y pautas que recogen. En este sentido, se ha realizado, en los últimos años, un 
gran esfuerzo para dotar a las bibliotecas de nuevos esquemas acordes a la realidad tecnológica. Los hitos más importantes son:

- 1998. Publicación de los FRBR, Functional Requirements for Bibliographic Records (Requisitos funcionales de los registros bibliográficos) que suponen el cambio más importante en el ámbito de la catalogación desde la publicación en 1971 de las ISBD. Contemplan, principalmente, tres componentes. En primer lugar, un modelo teórico para representar los conceptos y las relaciones. En segundo, un vocabulario para describir los niveles de representación y potencialmente, nuevas formas de visualizar la información en los OPACs.

- 2009 (marzo). Se aprueba el documento final de los FRAD, Functional Requirements for Authority Data (Requisitos funcionales para registros de autoridad), que se publicó en ese mismo año.

- 2009. Nueva declaración de principios internacionales de catalogación para su aplicación a los catálogos en línea de las bibliotecas (IFLA, 2009a). Esta declaración sustituye y amplía el alcance de los Principios de París siendo la primera vez que se tienen en cuenta pautas para la búsqueda y recuperación en entornos electrónicos. Se ha construido sobre la base de la tradición catalográfica existente y también sobre el modelo conceptual de la IFLA: FRBR y FRAD.

- En junio de 2010, se publica un nuevo código de catalogación denominado RDA, Resource Description and Access (Descripción y acceso de recursos) que va a sustituir a las AACR. Esta nueva norma se basa en los tres trabajos anteriormente citados. Y aúna los conceptos catalográficos con diferentes esquemas de codificación en los entornos electrónicos. Es decir, los catálogos que se construyan podrán realizarse con formato MARC o con metadatos o nuevas estructuras que puedan surgir.

Las AACR y, en general, los códigos catalográficos nacionales, son el reflejo de las bibliotecas y de las tecnologías de la década de los setenta y ochenta. Fueron pensadas para los catálogos en ficha, para los materiales impresos, y para usuarios con determinadas necesidades de información. Pasados más de treinta años, esa realidad ha cambiado sustancialmente. En 1988, un estudio realizado por el organismo responsable del desarrollo del formato MARC (International MARC Network) llamó la atención a las bibliotecas nacionales sobre el escaso tratamiento que se le brindaba a la información que se generaba en otros soportes que no fueran los impresos. Señaló la necesidad de adecuar los estándares para el tratamiento de estos nuevos soportes. Asimismo se recomendó mejorar la calidad de los registros bibliográficos buscando potenciar el intercambio y la cooperación entre las distintas instituciones (Estivill Rius, 2009).

Con el crecimiento vertiginoso de la información electrónica han nacido nuevos estándares para almacenar, recuperar y acceder a los objetos de información en un entorno de red. Estos nuevos estándares están relacionados, por una par- 
te, con prácticas más acordes con la tecnología existente y provienen fundamentalmente de otras iniciativas no puramente bibliotecarias como los lenguajes de marcas y los esquemas de metadatos.

Hoy en día, nos encontramos con colecciones virtuales compartidas por grandes consorcios, con usuarios habituados a interactuar en la Red, donde el catálogo tradicional de la biblioteca se convierte, a veces, en obstáculo más que en un facilitador del proceso de recuperación de la información. Dunsire (2007a) señala que frente a esta realidad, los metadatos utilizados para la descripción de los recursos de información necesitan adecuarse a la nueva variedad de soportes de información disponibles; y que estos deben poder abarcar las múltiples opciones, ya sea desde colecciones digitales hasta paquetes multicomponente orientados al aprendizaje.

Hace ya más de una década, algunos autores manifestaban que los OPACs no podían ser considerados como herramientas avanzadas ya que se sostenían sobre las prácticas tradicionales desarrolladas para los catálogos en fichas (OrtizRepiso y Moscoso, 1999). Estos catálogos no se adaptan a las necesidades de los actuales lectores, que están habituados a los nuevos entornos de búsqueda y recuperación de información que ofrecen las tecnologías web (Bianchi y Guerrini, 2009).

Esta situación ha producido un desfase entre el modelo que usamos y las tecnologías que disponemos, generando una brecha difícil de revertir si no se cambian las bases y principios utilizados para la catalogación.

El avance tecnológico que ha impactado en las formas de producción de la información, en el desarrollo de los catálogos de las bibliotecas, y en las nuevas demandas de los usuarios, ha sido el factor determinante para la elaboración de un nuevo código que diera respuesta a esta situación. Gestionar este cambio no es una tarea sencilla, ya que implica abordarlo de una forma interdisciplinar, donde se tendrán que considerar los aspectos bibliográficos, e informáticos entre otros (Bianchi, 2009). Debemos agregar, además, que las bibliotecas y los centros de documentación se caracterizaron por ser las únicas instituciones responsables de la organización del conocimiento y de la información en la época anterior a Internet. En la actualidad, sin embargo, se encuentran con nuevos competidores como son: las herramientas de búsqueda propias de la red, los proyectos de digitalización de fondos documentales que se desarrollan fuera del ámbito bibliotecario y las empresas comerciales, por ejemplo Amazon, que se presentan como oportunidades atractivas para los usuarios de información.

Así pues, las bibliotecas se encuentran en una situación que hace algunos años era impensable, compitiendo con organizaciones que tienen objetivos bien distintos a los establecidos para estas instituciones, pero que prometen satisfacer las mismas necesidades de los usuarios de las bibliotecas. Este contexto denota una necesidad ineludible de actualizar la normativa catalográfica, buscando ofrecer nuevas herramientas y mecanismos que satisfagan las necesidades de sus usuarios.

En el año 1997, la comunidad internacional de catalogadores, en la Conferencia Internacional sobre Principios y Desarrollo Futuro de las AACR celebrada en 
Toronto (Joint Steering Committee for Development of RDA, 1997), se planteó llevar a cabo una profunda actualización de la normativa catalográfica que tuviera un alcance internacional y que fuera coherente con la realidad tecnológica. El nuevo código catalográfico se publica en el mes de julio de 2010, después de más de diez años de trabajo.

Este nuevo código se presenta como el sucesor de las AACR y anuncia entre sus cambios el hecho de estar basado en un modelo conceptual y de haberse diseñado para el entorno digital (Oliver, 2010). La fuerte relación que tiene con el modelo conceptual FRBR se puede entender como la voluntad manifiesta de estar en sintonía con los desarrollos informáticos, ya que se definió utilizando el modelo entidad-relación para componer la realidad que se desea representar en las bases de datos. El nuevo código se estructura sobre las entidades y relaciones definidas por el modelo. Vamos a verlo con más detenimiento en el siguiente epígrafe.

\section{Los requisitos funcionales de los registros bibliográficos (FRBR)}

La Federación Internacional de Asociaciones de Bibliotecas e Instituciones (IFLA) a través del programa, ya extinguido, UBCIM (Universal Bibliographic Control and International MARC) decidió estudiar y analizar las funciones de los registros bibliográficos en relación a los diferentes media, aplicaciones informáticas y necesidades de los usuarios. Esta iniciativa, iniciada en el Seminario celebrado en Estocolmo en 1990, tomó cuerpo, ocho años después, con la publicación de los Functional Requeriments for Bibliographic Records: Final report (IFLA, 1998). La IFLA recomendó a las bibliotecas y agencias nacionales la adopción de los componentes del modelo como elementos básicos para el registro bibliográfico.

El estudio de los Requisitos Funcionales para los Registros Bibliográficos y su necesaria vinculación con los datos de autoridad, tanto para las entidades del segundo como tercer grupo del modelo FRBR, dio lugar a la realización de otros dos estudios, con similares características, que se materializaron en la definición de los modelos FRAD (Functional Requirements Authority Data) (IFLA, 2009b) y FRSAD (Functional Requirements for Subject Authority Data) (IFLA, 2010). Más de diez años después de los FRBR.

Estos modelos vienen a explicar de forma teórica lo que los Sistemas Integrados de Gestión de Biblioteca, ya habían resuelto en parte, de forma práctica, vinculando los registros bibliográficos, los de autoridades y los de localizaciones (holdings). Sin embargo, la definición de estos modelos brinda, a priori, nuevos elementos para comprender las relaciones que existen entre los datos que se consignan en estos registros. La clarificación de estas relaciones permitirá nuevas modalidades de procesamiento de la información que respondan a las necesidades planteadas por los usuarios y que estén en sintonía con los últimos desarro- 
llos tecnológicos. Supone, en principio, un paso más allá del formato MARC que se utiliza en la mayoría de los catálogos cuya estructura limita el establecimiento de relaciones y vínculos.

Las FRBR definen los objetivos principales de los catálogos: encontrar, identificar, seleccionar y acceder a los recursos de información del universo bibliográfico (Madison, 2005). Como ya explicamos en el epígrafe anterior, la función del modelo teórico es generar un marco de comprensión de lo que se debe describir en el universo bibliográfico. Por lo tanto, y mas allá de la recomendación realizada en la reunión de Copenhague, las bibliotecas no pudieron implementar el modelo FRBR, sino a través de la definición de modelos intermedios que establecieron relaciones de correspondencia entre el modelo FRBR y el utilizado para representar los datos (Le Boeuf, 2002). En general, por tanto, los catálogos que permiten visualizar la información según el modelo FRBR, deben extraer los datos de los tradicionales registros MARC. Zhang y Salaba (2009) señalan, entre las conclusiones de su investigación, la necesidad de que se desarrollen reglas de catalogación que estén en coherencia con el modelo para lograr su aplicación.

Algunas bibliotecas y comunidades afines han comenzado a experimentar y a investigar sobre los beneficios que podría aportar la aplicación de este modelo en la visualización de sus catálogos. Son experiencias en pleno desarrollo que todavía no han arrojado datos concluyentes como para justificar su adopción. Como ejemplos de implementaciones del modelo FRBR podemos citar a las bases de datos desarrollados por AustLit Gateway (Austlit, 2011), la experiencia de FindFiction de OCLC (2009a), el proyecto de la Universidad de Kent State (2011). Respecto a ejemplos de software que permite realizar visualizaciones nos podemos referir a: FRBR display tool (Library of Congress, 2009b), BIBSYS de la Biblioteca Nacional Noruega desarrollado en conjunto con la Universidad de Ciencia y Tecnología de este país (Aalberg y otros, 2006) y el sistema integrado de bibliotecas Virtua (VTLS, 2008).

Como se analizará, más adelante, las RDA adoptan este modelo de referencia teórico para establecer las instrucciones prácticas que permitan representar el universo según fue definido por las FRBR.

Tom Delsey es el actual editor de las RDA. Es posible que haya sido designado para ocupar este lugar, gracias a la relevancia que adquirió a partir del estudio que realizó sobre la estructura lógica de las AACR utilizando el modelo entidad/relación (Delsey, 1997; 1998; 1999). Asimismo, integró el grupo que definió el modelo FRBR. El conocimiento que adquirió sobre el modelo entidad relación a partir de estas dos experiencias es lo que probablemente explique la relación de las RDA con los modelos FRBR y FRAD.

Delsey había realizado, a finales de los noventa, un estudio en profundidad de las AACR con el objetivo de detectar sus incoherencias. Este le facilitó establecer las diferencias entre lo que determinó que eran las entidades del mundo real, pertenecientes al universo bibliográfico, y las entidades que representan una construcción bibliográfica utilizadas por las AACR. El punto de partida de este estudio fue su convencimiento de que la división de las AACR en dos partes 
(descripción y acceso) no era adecuada, ya que una hace referencia al ítem, y la otra a la obra. Términos, por otra parte, muy ambiguos, tal como se utilizaban en el código. Demostró que eran construcciones bibliográficas que no correspondían a las entidades encontradas en el universo bibliográfico real. Señala que el ítem puede equivaler a documento, colección, parte de un documento y hasta puede hacer referencia a un ejemplar. Estas inconsistencias generan problemas cuando se catalogan los distintos recursos, pero además, y fundamentalmente, dificultades para la actualización de las reglas. También concluyó que las reglas no reflejaban adecuadamente las relaciones que se presentaban entre los distintos recursos de información en el universo bibliográfico (Heaney, 2000).

Delsey (2009), partiendo del modelo FRBR propone una nueva forma de estructurar el código, que permite, sobre todo, incorporar los nuevos soportes y formas de contenido producto de la aplicación de las TIC, ofreciendo una coherencia interna en su estructura posibilitando una fácil actualización. Es en este punto, donde los modelos entidad relación definidos por IFLA realizan su aportación: dotar de una estructura lógica al nuevo código, prescindiendo de la organización deficiente que ofrecían las AACR basada en dos de las funciones que cumple un registro bibliográfico (descripción y acceso). El nuevo código se estructura sobre los tres grupos de entidades que se deben consignar en los registros bibliográficos (descripción, acceso a los datos bibliográficos y acceso por materias)

Es necesario destacar que, además de la estructura lógica, las RDA incorporan también la terminología, más adecuada al entorno tecnológico actual al referirse a las entidades y las relaciones que entre ellas se establecen. Esta estrecha relación nos obliga, para entenderlo mejor, a analizar el modelo FRBR. Se estructuran sobre 3 grupos de entidades que representan los objetos del universo bibliográfico real y las relaciones que se presentan entre ellas en función de las necesidades de los usuarios. Se parte, como sostiene Taylor, de la organización de los catálogos tradicionales, detectando sus defectos y proponiendo soluciones. En estos se definen, normalmente, distintos registros para una misma obra; el registro de la obra original, registros para otros formatos, distintas ediciones, traducciones, registros con obras nuevas que tenían como materia a la primera obra, como es el caso de las revisiones críticas, las adaptaciones, etc. (Taylor, 2007). Cada una de estas posibilidades se consigna en registros separados en el catálogo (en algunos casos afortunados, indicando sus vínculos a través de notas textuales o las posibilidades del formato MARC). Los OPACs carecen de una estructura lógica. Y esto, precisamente, es lo que el modelo FRBR quiere remediar.

Por tanto, el modelo conceptual, representa el universo bibliográfico real: diferentes entidades que se relacionan entre sí y que pueden describirse a través de distintos elementos o atributos. La entidad hace referencia a las cosas u objetos que debemos representar, los atributos son sus características, es lo que nos permite describirlas, y las relaciones son los vínculos o las interacciones que se producen entre ellas (Taylor, 2007).

Entidades, relaciones y atributos son las tres palabras clave en FRBR. Se definen 10 entidades que se ordenan en tres grupos diferentes. El primero, com- 
prende cuatro entidades básicas que son productos del esfuerzo intelectual o artístico (obra, expresión, manifestación e ítem). El segundo, las entidades responsables del contenido intelectual o artístico (persona y autor corporativo). Por último, el tercero, las que pueden servir de materia a una obra (concepto, objeto, acontecimiento y lugar, más todas las anteriores) (IFLA, 1998).

Con respecto al tipo de relaciones el modelo las agrupa en dos niveles; las relaciones generales y las relaciones específicas. Las primeras, de carácter amplio, se establecen entre las entidades de los distintos grupos, como, por ejemplo, entre la entidad obra del grupo 1 y la persona del grupo 2 que se relacionan porque la persona o la entidad corporativa del grupo dos es la creadora de la obra.

Las relaciones específicas se presentan entre las entidades de un mismo grupo, por ejemplo, entre dos expresiones de una misma obra, a partir de la traducción de una obra, o entre las distintas manifestaciones de una misma obra, como es el caso de las distintas ediciones. Podemos ver un ejemplo de estas relaciones en la figura 2.

\section{FIGURA 2}

Las entidades del Grupo 1 del modelo FRBR y las relaciones específicas

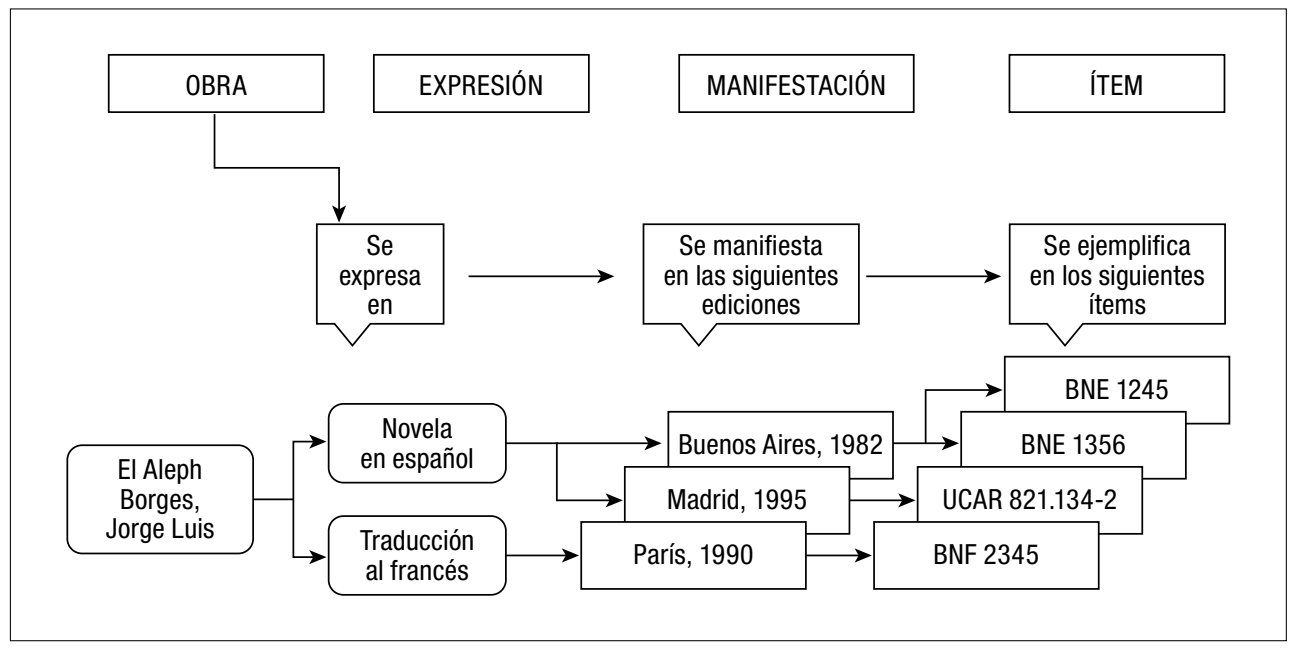

Fuente: Ortiz- Repiso, 2005.

Los tres grupos de entidades definidas en el modelo FRBR tienen relación con las tres actividades principales que cumple un registro bibliográfico. El primer grupo de entidades del modelo FRBR con la descripción (obra, expresión, manifestación e ítem). El segundo con los creadores, realizadores, productores y poseedores de las entidades del grupo 1 (personas y entidades corporativas), que 
darán acceso a los registros. El grupo 3 recoge las materias que puede abordar la entidad obra del grupo 1. Las entidades de este grupo se definen de la siguiente manera; concepto hace referencia a una idea o noción abstracta, por ejemplo Botánica. Objeto a una cosa material, incluye tanto objetos animados como inanimados, un objeto podría ser la camomila (planta medicinal). El evento es concebido como un acontecimiento o a una acción u ocurrencia, en este caso podríamos citar como ejemplo a una conferencia, una reunión, un período de tiempo. Y por último, lugar a una ubicación con el mas amplio alcance, puede ser terrestre, extraterrestre, histórica, contemporánea, geográfica, etc. (Taylor, 2007).

FIGURA 3

Funciones del registro bibliográfico

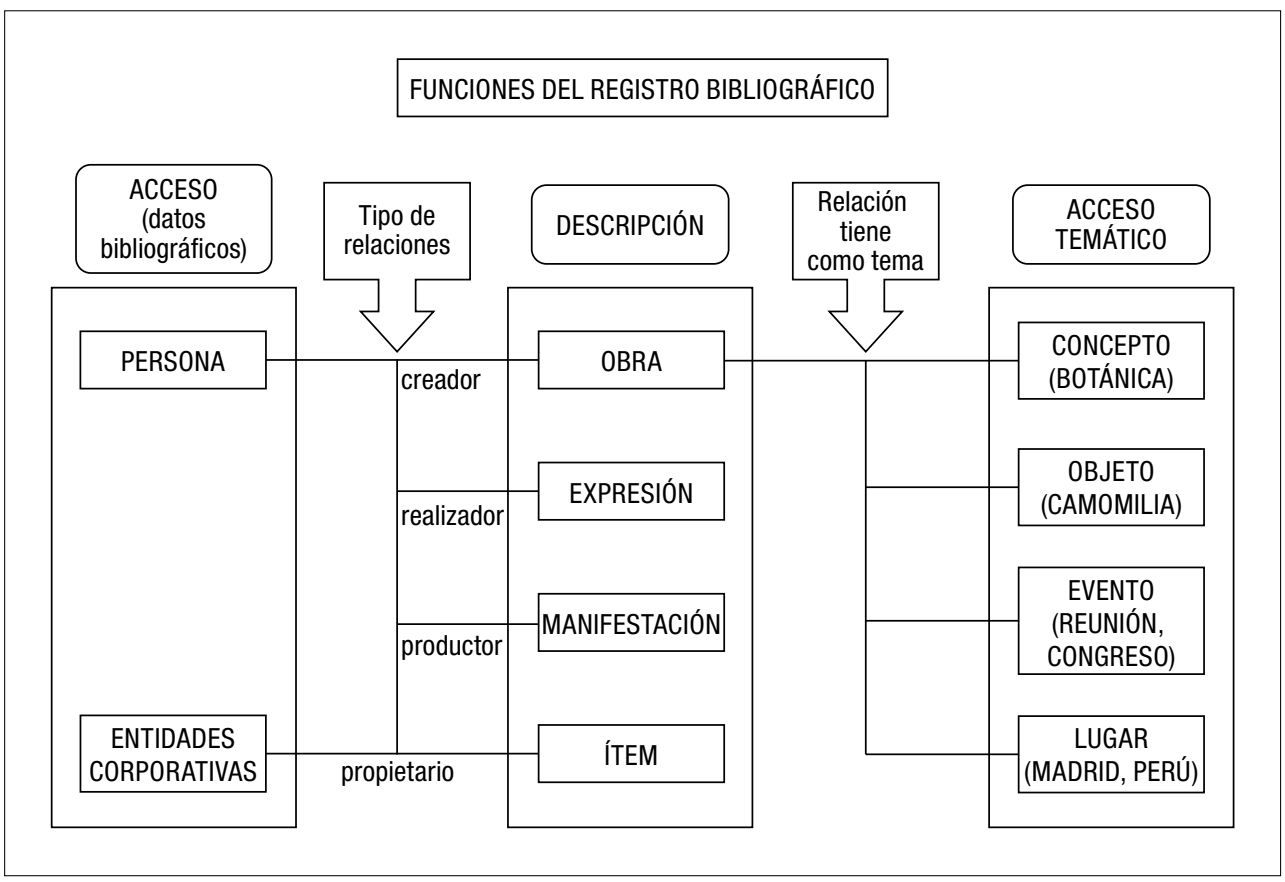

La figura 3 representa las tres funciones que cumple un registro bibliográfico y ubica a los tres grupos de entidades del modelo FRBR vinculadas con cada una de esas funciones. Este diagrama permite comprender la contribución del modelo: clarificar qué entidades se van a representar en un registro bibliográfico y establecer la relación entre estas y las funciones que deben cumplir en el mismo.

La pregunta que corresponde hacerse es y ¿cuál es el cambio, qué ventaja aporta? La definición del modelo FRBR hizo evidente la necesidad de definir un 
modelo complementario para el tratamiento de los datos de autoridad, reveló la necesidad de abordar los datos bibliográficos y de autoridad de forma coherente y en, cierto sentido, se alcanzaron avances significativos. Sin embargo, el modelo ofrece dudas a la comunidad bibliotecaria. Velucci (1997) sostiene que la definición del modelo no se realizó a partir de estudios empíricos que permitieran comprobar que las entidades identificadas, las relaciones, y las tareas de los usuarios que propone las FRBR estuvieran en consonancia con los intereses de los usuarios. En la misma línea Zhang y Salaba (2009) señalan la necesidad de realizar investigaciones que revelen la utilidad de los catálogos que permiten una visualización según las FRBR y la percepción que tienen los usuarios de esta nueva modalidad. Según Estivill Rius (2009), los proyectos y prototipos que se han realizado no han sido exhaustivos y han estado enfocados a recursos de información con características muy concretas, lo que no permiten sacar conclusiones que justifiquen su total adopción. Se acentúa, fundamentalmente, la necesidad de comprobar si las tareas de los usuarios según fueron definidas en el modelo FRBR están en consonancia con las modalidades en que los usuarios buscan la información (Velucci, 1997; Zhang y Salaba 2009).

Lo que podemos destacar por el momento es, sin duda, su gran aportación al establecimiento de una estructura lógica que da sustento al nuevo código donde se tratan a las entidades tal como se presentan en el universo bibliográfico. Y, además, donde se abordan en su conjunto, a través del análisis de sus relaciones. En definitiva será el tiempo y las investigaciones que se realicen las que nos permitirán comprobar la conveniencia de esta fórmula.

\section{El Código RDA: su estructura}

Como ya se ha señalado, la estructura del código se organiza según las entidades y relaciones del modelo FRBR y FRAD. Se divide en 10 secciones las cuales están precedidas por una introducción que explica el propósito y alcance del nuevo código, como sus objetivos y principios. Incluye una explicación de la nueva estructura del código, donde se indican los aspectos más relevantes que se encontrarán en cada sección. Se establecen cuales son los elementos básicos que hay que registrar cuando se describe un recurso, y a continuación se indica en qué sección se encuentran las instrucciones específicas para el registro de cada uno de ellos. En esta parte se incluyen algunos ejemplos para poder visualizar los cambios que el código propone. La introducción finaliza remarcando la desvinculación del código con cualquier formato de almacenamiento de datos. Pero, sin embargo, para facilitar la comprensión de los cambios, se incluyen ejemplos de acuerdo a las normas ISBD y al formato MARC21 en el apéndice D.

La tabla 1 nos permite comparar la estructura y su relación con los modelos FRBR y FRAD. Los capítulos de la Sección 1 a 4 están dirigidos a la descripción de las distintas entidades de los dos modelos ya citados, y del 5 en adelante al registro de las relaciones. Cada sección empieza con un capítulo donde se inclu- 
TABLA I

\section{Estructura de las RDA}

\begin{tabular}{l|l}
\hline Introducción & $\begin{array}{l}\text { Propósito y alcance. Objetivos y principios. } \\
\text { Estructura. Elementos básicos (core elements). Puntos } \\
\text { de Acceso. Ejemplos y codificación de los datos a } \\
\text { partir de las RDA. }\end{array}$
\end{tabular}

\begin{tabular}{l|c}
\hline $\begin{array}{l}\text { SECCIÓN 1-4. REGISTRO DE ATRIBUTOS } \\
\begin{array}{l}\text { Sección 1. Manifestación-ítem (Entidades } \\
\text { del modelo FRBR) }\end{array}\end{array}$ & $\begin{array}{c}\text { Instrucciones generales para registrar los atributos de } \\
\text { las manifestaciones y de los ítems. }\end{array}$ \\
$\begin{array}{l}\text { Sección 2. Obra-expresión (Entidades del } \\
\text { modelo FRBR) }\end{array}$ & $\begin{array}{c}\text { Instrucciones generales para registrar atributos de la } \\
\text { obra y expresión. Identificación de las obras y } \\
\text { expresiones. Descripción del contenido. }\end{array}$ \\
$\begin{array}{l}\text { Sección 3. Persona, familia y entidad } \\
\text { corporativa (Entidades del modelo } \\
\text { FRAD) }\end{array}$ & $\begin{array}{c}\text { Instrucciones generales para registrar los atributos de } \\
\text { las personas, familias y entidades corporativas. } \\
\text { Identificación de las persona, familias y de las entidades } \\
\text { corporativas. }\end{array}$ \\
\hline $\begin{array}{l}\text { Sección 4. Concepto, objeto, evento y } \\
\text { lugar. (Entidades del modelo FRBR) }\end{array}$ & $\begin{array}{c}\text { Instrucciones generales para el registro de los atributos } \\
\text { y la identificación de los conceptos, objetos, eventos } \\
\text { lugares. Se incluye solo el capítulo general y el } \\
\text { referido a la identificación de los lugares, los otros } \\
\text { se encuentran en desarrollo. }\end{array}$ \\
\hline
\end{tabular}

\section{SECCIÓN 5-10. REGISTRO DE RELACIONES}

\begin{tabular}{c|c}
$\begin{array}{c}\text { Sección 5. Primarias entre la obra, expresión, } \\
\text { manifestación y el ítem (Relaciones del } \\
\text { modelo FRBR) }\end{array}$ & $\begin{array}{c}\text { Instrucciones generales para el registro de las relaciones } \\
\text { primarias. }\end{array}$ \\
\hline $\begin{array}{c}\text { Sección 6. Personas, familias y entidades } \\
\text { corporativas (Relaciones definidas por } \\
\text { el modelo FRAD) }\end{array}$ & $\begin{array}{c}\text { Instrucciones generales para el registro de las relaciones } \\
\text { asociadas con una persona, familia, entidad corporativa } \\
\text { con un recurso. } \\
\text { Relación entrelas personas, familias, entidades corporativas } \\
\text { con una obra, expresión, manifestación e ítem. }\end{array}$ \\
\hline $\begin{array}{c}\text { Sección 7. Hacia los conceptos, objetos, } \\
\text { eventos y lugares. (Relaciones definidas } \\
\text { por el modelo FRBR) }\end{array}$ & $\begin{array}{c}\text { Capítulo 23. Instrucciones generales para el registro del } \\
\text { tema de una obra (Capítulo que se encuentra en } \\
\text { desarrollo). }\end{array}$ \\
\hline $\begin{array}{c}\text { Sección 8. Entre las obras, expresiones, } \\
\text { manifestaciones e ítems. (Relaciones } \\
\text { primarias del modelo FRBR) }\end{array}$ & $\begin{array}{c}\text { Instrucciones generales para el registro de las relaciones } \\
\text { entre las obras, expresiones, manifestaciones e ítems. } \\
\text { Relaciones entre obras, expresiones, manifestaciones, }\end{array}$ \\
\hline $\begin{array}{c}\text { Sección 9. Entre las personas, familias y } \\
\text { las entidades corporativas. (Relaciones } \\
\text { definidas por el modelo FRAD) }\end{array}$ & $\begin{array}{c}\text { Instrucciones generales para el registro de las relaciones } \\
\text { entre personas, familias, y entidades corporativas. } \\
\text { Entre personas, familias, y entidades corporativas. }\end{array}$ \\
\hline $\begin{array}{c}\text { Sección 10. Entre los conceptos, objetos, } \\
\text { eventos y lugares. (Relaciones definidas } \\
\text { por el modelo FRBR) }\end{array}$ & $\begin{array}{c}\text { Capítulo 33. Instrucciones generales para el registro de } \\
\text { las relaciones entre conceptos, objetos, eventos y } \\
\text { lugares (capítulos en desarrollo.) }\end{array}$ \\
\hline
\end{tabular}


yen instrucciones generales que son de referencia para los otros específicos. En ellos se describen las entidades y las relaciones que se generan entre ellas.

Esta estructura implica un cambio en la forma de utilización respecto a las AACR. Para la descripción de un recurso será necesaria la consulta a varios capítulos, y el suficiente conocimiento del mismo para poder ubicar las instrucciones específicas. A modo de ejemplo, para registrar los puntos de acceso deberemos consultar varios capítulos, a diferencia de las AACR que reunían todas las reglas referente a este aspecto en el capítulo 21. Otro cambio destacable es que los datos que se pueden registrar como notas, según la tradición de las AACR, están en el nuevo código asociados a cada elemento específico de la descripción. Dentro de las instrucciones dirigidas al registro del elemento título, se encuentran las que indican cuando se debe generar una nota. Esto señala que deberemos cambiar nuestras dinámicas de trabajo y establecer los recorridos por las distintas secciones y capítulos para la descripción de los recursos de información. La versión electrónica del Código incluye una serie de flujos de trabajo sugeridos para la descripción de los distintos recursos y vínculos entre las reglas de las AACR y las instrucciones del nuevo código (RDA Toolkit, 2010).

En la introducción se incluye un esquema que facilita la ubicación dentro de los capítulos de las instrucciones específicas para cada uno de los elementos que se deberá registrar. Seguramente no deberemos echar mano a estas herramientas hasta que estemos lo suficientemente familiarizados con esta nueva aproximación.

\section{Los cambios en la generación de los registros bibliográficos a partir de la aplicación de las RDA}

La pregunta que cabe formularse ahora es qué significará la aplicación de estas nuevas reglas o instrucciones en la generación de los registros bibliográficos. Una de las primeras cosas que debemos destacar, es que las RDA se presentan como un estándar de descripción o de representación de datos independiente de cualquier estándar de almacenamiento. Es decir, que no prescribe la forma en que se deberá presentar el registro bibliográfico dejando a la libre decisión de las bibliotecas. Pero la verdadera razón es que el código pueda ser utilizado tanto en los catálogos y en sistemas de gestión tradicionales, como en nuevos modelos que se desarrollen. Los ejemplos en el código no siguen ninguna estructura preestablecida para la presentación de los datos, pero en el Apéndice D los ejemplos que se incluyen se muestran según las ISBD y el formato MARC21.

Sabemos que el nuevo código, si bien pretende desvincularse de los estándares de almacenamiento de la información, tiene que tener cierta relación con los mismos, y de hecho la tiene. Esto puede observarse en el mencionado apéndice y en los distintos escenarios de implementación que definió Delsey (2009) donde establece las distintas formas que podrán adoptar los registros y catálogos. 


\section{Primer escenario de implementación de las RDA}

Delsey define el primer escenario a partir de la utilización de una base de datos relacional u orientada a objetos, definida según las entidades de los modelos conceptuales FRBR y FRAD. Se crearían registros para cada una de las entidades del grupo 1 (obra, expresión, manifestación e ítem). Los puntos de acceso estarían en registros creados para las entidades primarias del modelo FRAD y se establecerían los vínculos con los datos descriptivos de las entidades del modelo FRBR a través de hiperenlaces. Este modelo no solo simplificaría la catalogación sino que sería coherente con el modelo de la web semántica o linked data al cual nos referiremos al finalizar este epígrafe.

\section{FIGURA 4}

Nuevo modelo de representar los datos bibliográficos

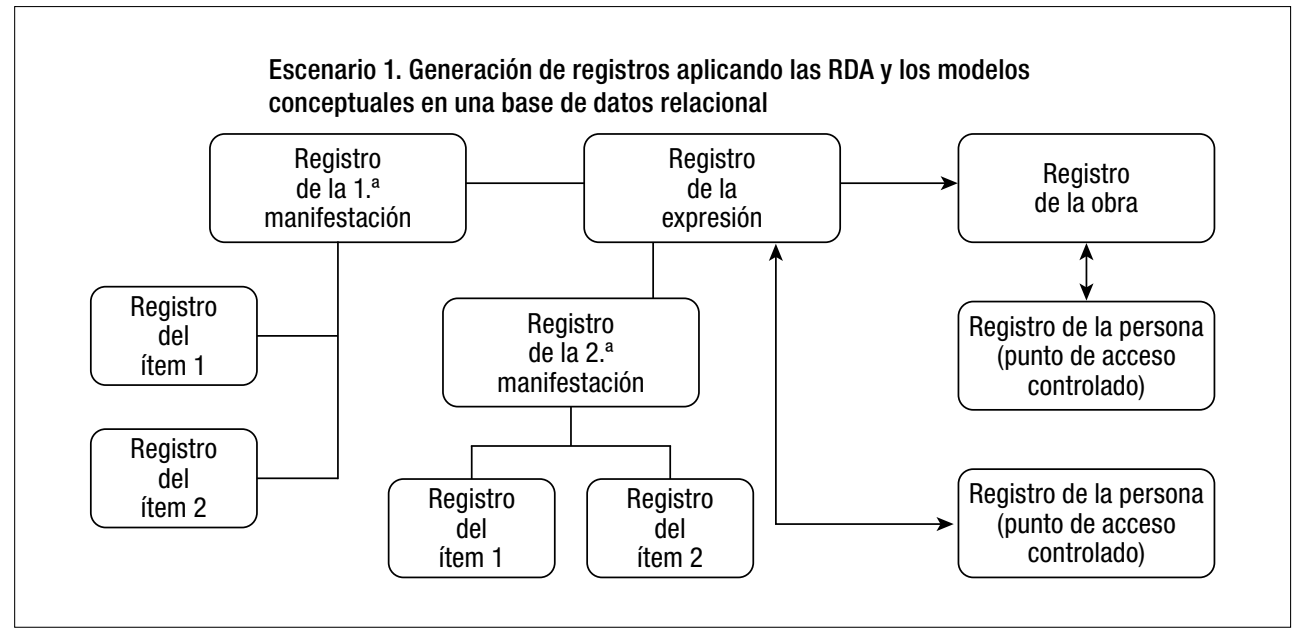

La figura 4 muestra una serie de registros que representan a las distintas entidades del modelo FRBR, se incluyen registros para la obra, expresión, manifestación, ítem (entidades del grupo 1) y persona (entidad del grupo 2). Podemos observar que todos ellos se conectan a través de las relaciones que se generan entre estas entidades y que fueron definidas en el modelo FRBR. Con líneas simples están marcadas las relaciones entre las entidades del grupo 1 y con flechas las relaciones entre las entidades del grupo 1 y las del grupo 2.

\section{Segundo y tercer escenario de implementación}

En estos dos casos, los registros se almacenarían en las bases de datos utilizadas tradicionalmente en los Sistemas Integrados de Gestión de Biblioteca. En el segundo, se obtendrían registros de autoridad y bibliográficos enlazados a 
El contenido es un atributo de la entidad expresión. La instrucción 6.9 del nuevo Código lo define como «una categorización que refleja la forma principal de comunicación en la que el contenido es expresado y percibido por los sentidos bumanos" Por ejemplo, imagen, texto, notación musical entre otros más (RDA, 2010).

Por tipo de medio se entiende según la instrucción 3.2 «la categorización que refleja el tipo de intermediación electrónica requerida para acceder al contenido del recurso de información". Ejemplo: audio, computadora, microforma, no mediado para el caso que no requiera ningún instrumento de intermediación para el acceso (RDA, 2010).

La instrucción 3.3 define al tipo de portador como "la categorización que refleja el formato de almacenamiento y soporte que adquiere un portador según el tipo de intermediación electrónica que requiera para brindar acceso al contenido de un recurso de información". A tales efectos lo tipifica como portadores de audio, portadores de ordenadores, portadores de microformas, etc.

Con la definición de estos tres atributos se supera el problema que presentaba la asignación de la designación general del tipo de material (DGM) en las AACR que solapaba contenidos y material físico, haciendo muy difícil su aplicación. Hider (2009) y Salamanca Chiverto (2008) sostienen que las DGM y los capítulos de la primera parte del Código AACR se presentaban como categorías excluyentes, la práctica demostró que dos y hasta tres capítulos se podían utilizar para la descripción de un mismo recurso. Esta situación le planteaba al catalogador la dificultad de optar por el capítulo más adecuado. ¿Qué capítulo utilizar cuando se encontraba, por ejemplo, con un mapa en soporte digital?: ¿el que refería a cartografía o el dirigido a recursos electrónicos?

La propuesta del código apunta a resolver este viejo conflicto y, además, a mejorar las posibilidades de recuperación de información, ya que estos atributos permitirán establecer algoritmos de búsqueda dirigidos exactamente a lo que el usuario necesita. Para registrar estos tres atributos las RDA proponen la utilización de un vocabulario controlado que fue definido en conjunto con el estándar Online Information Exchange (ONIX) utilizado por las editoriales para la descripción del contenido de sus productos (Dunsire, 2007a).

No nos proponemos hacer una enumeración exhaustiva de los cambios prácticos propuestos por el nuevo código, queremos, únicamente, señalar los más destacables. Como son, por ejemplo, la definición de los elementos básicos que se deben registrar para describir un recurso de información. Estos sustituyen a los elementos obligatorios que eran utilizados en el primer nivel de descripción de las AACR.

Recordamos que el nuevo código tiene una estructura completamente distinta como ya hemos analizado, y que surge de las bases conceptuales que le otorgan los modelos FRBR y FRAD. En sintonía con los modelos, y buscando priorizar el interés del usuario, en cada sección del Código se describen los objetivos que alcanza cada una de ellas en relación con las necesidades de los usuarios. 
En los escenarios de implementación tradicionales, que requieren el uso del formato MARC21, se crean nuevos registros bibliográficos cuando hay un cambio en el modo de publicación, en el tipo de medio, en el título y mención de responsabilidad de una publicación seriada y en la mención de edición. Para el caso de las monografías que se publican en más de un volumen, cuando se presenten cambios en el modo de publicación y en el tipo de medio. El hecho de habilitar la creación de un nuevo registro a partir de un cambio en el tipo de medio, permite resolver la situación que se planteaba en las AACR cuando teníamos la misma obra publicada en forma impresa y digital.

Existen cambios en las fuentes de información, en el nuevo código se las denomina fuentes preferidas de información y establece mecanismos más flexibles para extraer los datos que se van a consignar. Se elimina el uso de corchetes prescripto por las AACR cuando la información no se extrae de las fuentes prescriptas, este recurso carecía de interés para los usuarios y muchos catalogadores.

Desde nuestro punto de vista los verdaderos cambios en los registros bibliográficos se podrán observar a partir de las nuevas estructuras que se desarrollen. En este sentido, la comunidad catalográfica ha demostrado mucho interés en el acercamiento entre la comunidad Dublin Core que se inició en el año 2007, con ocasión de una reunión celebrada en la British Library. Fue así que se conformó un grupo de trabajo que se denominó DCMI/RDA Task Group cuyo trabajo fundamental fue la compatibilización de los lenguajes controlados definidos por el nuevo código en RDF, un estándar de la web semántica que permitirá desarrollar aplicaciones informáticas que utilicen Dublin Core compatibles (Hillmann y otros, 2010). Seguramente esta alianza será la que posibilitará generar nuevas modalidades de estructurar y almacenar los registros bibliográficos que sean más acordes a las tecnologías de vanguardia. El trabajo que se viene realizando con el estándar RDF, que permite que los datos bibliográficos sean abiertos y salgan del los circuitos exclusivos utilizados por la comunidad bibliotecaria (como es el caso del formato MARC), permite que se realicen vínculos flexibles de datos (linked data) y, se enriquezca, de esta forma, la información que se ofrece. Los Encabezamientos de materia para bibliotecas públicas recientemente disponibles en linked open data o los de la Bibliografía inglesa y francesa, son buen ejemplo de lo que estamos hablando. Agenjo y Hernández (2010) señalan que tanto la web semántica como la comunidad bibliotecaria comparten el interés por identificar la información de forma unívoca, utilizar los términos de forma no ambigua, y establecer relaciones y referenciar las mismas. Por lo tanto el trabajo conjunto en esta línea puede ser prometedor y terminar en el desarrollo de herramientas de búsqueda y recuperación de información más eficientes que los catálogos tradicionales.

La vinculación de la comunidad RDA con Dublin Core ofrece la posibilidad de superar las limitaciones que tiene el formato MARC. Contar con un nuevo estándar permitiría, a su vez, aumentar el intercambio de información entre distintas comunidades así como facilitar la generación de registros de forma auto- 
mática. Hasta el momento se han hecho avances importantes aunque el trabajo no está aún concluido. En el sitio web del grupo DCMI/RDA (2011) se anuncia que en la próxima conferencia Internacional sobre Dublin Core se presentará el informe final sobre la representación de los elementos y vocabularios controlados del nuevo código RDA en el estándar RDF. Los avances logrados se pueden observar en el Open Metadata Registry (2011), sitio en el que se ha ido registrando los términos del nuevo código vinculándolos a un identificador a través de un URL. A modo de ejemplo se puede consultar el atributo "tipo de portador", donde hay una nota de alcance que explica la aplicación del término, la comunidad que lo utiliza, en este caso las bibliotecas y servicios de información, el idioma y un URI (Universal Resource Identifier) que permite su identificación de forma inequívoca. El URI, como es sabido, es uno de los principales actores del Linked data (Heath y Bizer, 2011).

El desarrollo realizado con el estándar Dublin Core, junto con la conformación del grupo de trabajo del Consorcio Web para Linked Data denominado W3C Library Linked Data Incubator Group, cuyo objetivo es incrementar la interoperabilidad de los datos en la web, especialmente dentro del ámbito de la comunidad bibliotecaria, auguran importantes cambios en los sistemas informáticos y en la forma de estructurar e intercambiar la información (W3C Library Linked Data Incubator Group, 2010). Y hace patente, la necesidad, cada vez mayor, de una renovación de los Sistemas Integrados de Gestión de Bibliotecas.

La gran fortaleza que presenta esta iniciativa es el alto potencial que ofrece la utilización de las URI para establecer enlaces entre los datos de la web. Una cosa positiva es que las RDA definen para sus entidades a los identificadores como atributos, y es por lo tanto plausible la utilización de las URI. A través de ellas se podría fácilmente establecer las relaciones que se definen en los modelos conceptuales que dan sustento al nuevo código. Creemos que este es el camino que hay que tomar para producir un verdadero cambio en las prácticas catalográficas; no podemos seguir apostando por los formatos tradicionales que se generaron en otra época y que el desarrollo tecnológico revela su obsolescencia. Como dicen Peset y colaboradores La oportunidad de participar en la web semántica puede abrir las puertas de nuestra comunidad al mundo web o hacernos perder el tren de las tendencias mundiales (Peset y otros, 2011).

Estivill (2011) señala que posiblemente cuando las RDA funcionen en el entorno de la web semántica podremos realmente evaluar los beneficios que ofrece, sobre todo en lo que refiere al intercambio de información con otras comunidades no bibliotecarias. Enlazar datos que sumen valor a la información es el objetivo prioritario.

Desde nuestro punto de vista, los resultados que se presenten en la próxima conferencia de Dublin Core serán claves para poder visualizar algunas de estas nuevas modalidades para registrar la información utilizando las RDA y el lenguaje RDF. Constituirá un buen observatorio sobre los beneficios que ofrece el nuevo código y la utilización de los nuevos parámetros tecnológicos. 


\section{Las bibliotecas y la implementación del nuevo código}

La pregunta fundamental que debemos hacernos ahora es cómo se va a implementar este código y quienes lo van a adoptar. Tal vez sea todavía muy pronto para poder responder a estos interrogantes, el código fue publicado a mediados del 2010, y la Library of Congress es la encargada de coordinar la evaluación del mismo. Para ello definió un plan de trabajo que involucra a las otras dos bibliotecas nacionales del país, la National Agricultural Library y la National Library of Medicine. A su vez, hay otras instituciones norteamericanas que forman parte del equipo de evaluación del nuevo código. El plan contempla un calendario bastante ajustado, que comenzó en julio y terminó el 9 de mayo de 2011, con la publicación del Report and Recommendations of the U.S. RDA Test Coordinating Committee (U.S. RDA, 2011) informe final que es decisivo para la transición no solo de los países angloamericanos, sino también de los europeos como podremos comprobar en el cuadro que se incluye más adelante.

La evaluación se llevó adelante con 26 instituciones norteamericanas que elaboraron entre 20 y 25 registros bibliográficos utilizando las RDA y las AACR2 para realizar comparaciones respecto a las dificultades que podría generar la adopción del nuevo código. Pero para ampliar la cobertura y, sobre todo, comprobar la utilidad del nuevo código para otras comunidades no bibliotecarias, se aseguró la participación de instituciones que utilizan otras normas como es el caso de la AMIM (Archival Movind Image Materials), DACS (Describing Archives: A Content Standard), CCO (Cataloging Cultural Objects), entre otros.

El Comité de Evaluación se centró en los siguientes aspectos: evaluación del nuevo código en el entorno bibliotecario e informático actual, en los aspectos técnicos y operativos, y en las inversiones financieras que podría implicar la adopción del mismo. Asimismo se evaluaron los beneficios que ofrece a los bibliotecarios y usuarios finales.

El informe concluye recomendando la adopción del nuevo código por parte de las tres bibliotecas nacionales de Estados Unidos a partir de enero de 2013, una vez que las recomendaciones surgidas en el marco de la evaluación sean incorporadas al mismo.

Una de las mayores debilidades que demostró la evaluación es que el nuevo código no ofrece beneficios inmediatos si se implementa sobre los sistemas tradicionales de almacenamiento de información. En el ejemplo de formato MARC que se incluye en la Figura 5 se observan que los cambios son mínimos. El hecho de que el código sea independiente de cualquier formato de almacenamiento es, a priori, una ventaja, pero no tener hasta el momento una alternativa que permita implementar el primer escenario descripto por Delsey, es una limitación para poder concluir definitivamente sobre su potencial. Desde nuestro punto de vista este ha sido un punto álgido en la evaluación del código y lo vinculamos al anuncio realizado recientemente por la Library of Congress de dar comienzo al desarrollo de una nueva infraestructura que permita migrar los registros MARC a un nuevo sistema (Library of Congress, 2011). 
En el informe se destaca, además el interés de que el nuevo código pueda ser utilizado en los distintos entornos donde se produce información, ya sea entre las bibliotecas u otros productores de información. Uno de los hallazgos de la evaluación, es que el código no conforma aún a los posibles usuarios especializados como es el caso de la comunidad de la imagen en movimiento y grabaciones sonoras que señalaron debilidades en el capítulo 17 del código. La comunidad de publicaciones seriadas está también trabajando para elevar propuestas de cambios al JSC.

Se señalaron también dificultades en el establecimiento de las relaciones, la evaluación demostró que los catalogadores presentaron dificultades en identificar correctamente las entidades obra y expresión al momento de realizar el registro.

También se destacó el interés de que el nuevo estándar permita mayor flexibilidad en el entorno digital, que mejore las capacidades para la descripción de otros soportes no tradicionales y que permita generar metadatos utilizando las tecnologías del entorno linked data. Una cuestión interesante es que se manifiesta preocupación porque el código sigue mostrando una fuerte vinculación a las prácticas anglosajonas. Este último aspecto es importante, desde nuestro punto de vista, para que el nuevo código sea adoptado por otras comunidades de forma generalizada. La preparación del código encaminada por los cuatro países anglosajones y la evaluación realizada por bibliotecas de los Estados Unidos, limita mucho la propuesta original de desarrollar un código de alcance internacional.

Respecto a lo que sucederá fuera de la comunidad anglosajona podemos adelantar que en agosto de 2010 se celebró en Europa un encuentro que se tituló "RDA in Europe: making it happen» (RDA in Europe, 2010) donde varias bibliotecas nacionales de los países europeos presentaron su situación actual respecto a la catalogación y cuáles son las perspectivas de adopción del nuevo código. Resumimos los resultados en la tabla II.

Como podemos observar hay varios países que están considerando adoptar el nuevo código, pero en la mayoría de los casos, se requiere de la traducción del mismo al idioma nacional. Noruega y Suecia participaron activamente de la revisión del borrador final, la Biblioteca Nacional de España, ha seguido muy de cerca todo el proceso y ha implementado su propia instancia de evaluación, pero todavía no tiene una decisión final, espera también los resultados de las Bibliotecas Nacionales de los países miembros de las JSC y señala la necesidad de una traducción al español. Italia es el país que posiblemente se encuentre más lejos de adoptar el nuevo Código, ya que ha publicado el suyo propio en el año 2009. Observamos como uno de los mayores obstáculos el problema lingüístico, hubiera sido deseable que si el JSC se planteó como objetivo elaborar un código de alcance internacional, que lo hubiera publicado simultáneamente en varias lenguas (Picco, 2007).

¿Qué pasará con el resto de los países?, es una buena pregunta, quienes adoptarán el nuevo código y cómo lo harán no podemos todavía establecerlo, pero si podemos anunciar que si OCLC define dentro de su política de catalogación cooperativa la adopción de este nuevo estándar, estará forzando la situación. 
TABLA II

Situación del código RDA en algunas Bibliotecas Nacionales de Europa

\begin{tabular}{|c|c|c|}
\hline País & $\begin{array}{l}\text { Estado actual de la catalogación } \\
\text { respecto a la adopción de estándares }\end{array}$ & Situación respecto a las RDA \\
\hline Dinamarca & $\begin{array}{l}\text { Reglas propias basadas en AACR. } \\
\text { Formato basado en UKMARC con } \\
\text { adiciones del formato MARC21 }\end{array}$ & $\begin{array}{l}\text { Necesidad de evaluar el nuevo código. } \\
\text { Se necesita la traducción al danés. Se } \\
\text { están esperando los resultados del } \\
\text { proceso de evaluación de la Library of } \\
\text { Congress. }\end{array}$ \\
\hline Noruega & $\begin{array}{l}\text { Traducción de las AACR adaptado a } \\
\text { las políticas nacionales. Formato } \\
\text { basado en MARC21 }\end{array}$ & $\begin{array}{l}\text { Se participó del proceso de revisión del } \\
\text { borrador final de las RDA. } \\
\text { Para implementar nuevo Código es } \\
\text { necesario adoptarlo junto con Suecia } \\
\text { y Dinamarca. }\end{array}$ \\
\hline Suecia & $\begin{array}{l}\text { Traducción de las AACR. Formato } \\
\text { MARC21. }\end{array}$ & $\begin{array}{l}\text { Se participó del proceso de revisión del } \\
\text { borrador final de las RDA. } \\
\text { Necesidad de hace traducción al Sueco. } \\
\text { Decisión final dependerá de los } \\
\text { resultados que arrojen proceso de } \\
\text { evaluación llevado a cabo por la } \\
\text { Library of Congress. }\end{array}$ \\
\hline Finlandia & AACR y formato MARC21. & $\begin{array}{l}\text { Mucho interés en el nuevo Código. Se } \\
\text { formó un grupo que está trabajando } \\
\text { sobre la evaluación de las RDA en la } \\
\text { Biblioteca Nacional. }\end{array}$ \\
\hline Suiza & AACR y formato MARC21. & $\begin{array}{l}\text { Se necesita la traducción del código al } \\
\text { francés, alemán e italiano. } \\
\text { Colaboración entre Francia y Alemania. } \\
\text { Esperar resultados del proceso de } \\
\text { evaluación llevado por la Library of } \\
\text { Congress. }\end{array}$ \\
\hline Italia & $\begin{array}{l}\text { Reglas propias publicadas en el año } \\
\text { 2009. UNIMARC. }\end{array}$ & $\begin{array}{l}\text { Se necesita la traducción de las RDA al } \\
\text { italiano. }\end{array}$ \\
\hline Portugal & $\begin{array}{l}\text { Reglas nacionales necesitan ser } \\
\text { actualizadas. Formato UNIMARC }\end{array}$ & $\begin{array}{l}\text { Se va a formar un grupo de evaluación } \\
\text { a nivel nacional próximamente. } \\
\text { Traducción al portugués. } \\
\text { Necesidad que las RDA contemplen las } \\
\text { políticas nacionales. }\end{array}$ \\
\hline España & $\begin{array}{l}\text { Desde el año } 2007 \text { se está evaluando } \\
\text { cambios en el Código nacional. } \\
\text { Reglas de Catalogación Españolas y } \\
\text { formato MARC21. }\end{array}$ & $\begin{array}{l}2009 \text { se realiza un estudio sobre las RDA, } \\
\text { se produce un documento que señalan } \\
\text { los ajustes que necesita el formato } \\
\text { MARC } 21 \text { para poder evaluar el código. } \\
\text { Evaluar traducción del código. } \\
\text { Para tomar una decisión final se va a } \\
\text { esperar resultados de implementación } \\
\text { de los países anglosajones. }\end{array}$ \\
\hline
\end{tabular}


La globalización y la participación en proyectos cooperativos dejan muy poca autonomía a las bibliotecas, si quieren hacer parte de la red deberán tener que aceptar las nuevas reglas de juego.

\section{Conclusiones}

El mundo de los catálogos, en la actualidad, está sufriendo numerosos cambios para poder acompasar el ritmo tecnológico imperante. Nos encontramos, en este momento, en una etapa de transición en la que las normas y formatos tradicionales siguen empleándose de forma mayoritaria. Pero comienzan, al mismo tiempo, a materializarse nuevas formas para los catálogos que, con seguridad, se consolidarán en los próximos años. Este cambio es absolutamente necesario, ya que la catalogación está marcada por el entorno manual en el que fue creándose y desarrollándose. Ahora es tiempo de adaptar las prácticas antiguas a un nuevo medio para que el catálogo siga representando un papel importante entre el gran número de recursos de información a los que tenemos acceso.

Respecto a las RDA debemos señalar que van a producir un cambio muy significativo en la forma de trabajo del catalogador, la nueva estructura basada en las FRBR y FRAD obliga a cambiar las dinámicas tradicionales de trabajo. Conduce a incorporar una nueva terminología y forma de pensar al momento de catalogar. Con respecto a la creación de los registros bibliográficos, si se sigue utilizando el formato MARC21, los cambios que se introducen son mínimos. Ahora, la gran incertidumbre se plantea en las nuevas modalidades de implementación, que podrán adquirir éstos a partir de los desarrollos que surjan con la utilización de las herramientas de la web semántica, pero que, en todo caso, es todavía muy pronto para concluir al respecto.

Por otro lado, parecería que las RDA no responden de forma adecuada al planteamiento de simplificar la catalogación, los resultados a priori que arroja esta investigación hacen presumir que deberán ser utilizadas por catalogadores con mucha experiencia, alejándose cada vez más de otras comunidades que podrían interesarse en su aplicación, como son los editores, archivos, museos, y así poder ampliar el universo de cooperación.

Después del estudio realizado, nos preguntamos si el modelo vigente de la catalogación, que propone dar respuesta desde un único código al variado universo bibliográfico es el adecuado. O si tal vez, la variedad de recursos y sus características intrínsecas requieren varias herramientas que contemplen de forma específica el tratamiento de cada uno de ellos, dando respuestas más sencillas y flexibles. Y que, por último, que sean las tecnologías las que desarrollen mecanismos que permitan subsanar lo que los estándares de descripción no pueden hacer. En esta línea orientaremos nuestra investigación, para aportar en esta necesaria discusión que debemos abordar en la comunidad de catalogadores.

Por último, no podemos concluir sin hacer referencia a los desafíos tecnológicos que implica adoptar el nuevo código. Como ya lo mencionamos, las RDA 
son independientes de las normas de almacenamiento y presentación de datos, brindándoles a las instituciones la posibilidad que adopten los mecanismos que sean más adecuados a sus necesidades. Pero esta libertad es bastante limitada por dos razones; la primera es la disposición real de tecnologías que sean coherentes con el planteamiento del código, mencionamos como ejemplo que se definen elementos básicos para describir cualquier recurso, y por lo tanto, en algún lugar, se deben registrar: la incorporación de los campos 336, 337 y 338 son solo una muestra.

La segunda limitación tiene que ver con la compatibilidad de los registros que se generen en modelos nuevos, es decir, tiene que ser un instrumento que permita intercambiar la información con las demás bibliotecas, especialmente en un momento de transición, en donde cada institución lo hará a su ritmo, según sus posibilidades y "creencias", lo que provocará que convivan simultáneamente registros generados a partir de las AACR con los nuevos según las RDA.

Delsey (2009) definió los tres posibles escenarios de implementación; el segundo y el tercero corresponden a los catálogos disponibles en la actualidad, pero el primero es toda una incertidumbre. El gran desafío que tenemos por delante es interesar a la comunidad informática en que se involucre en proyectos de desarrollo de software con las características definidas en ese nuevo escenario.

\section{Bibliografía}

Aalberg, T.; Berg Haugen, F., y Husby, O. (2006). A tool for converting from MARC to FRBR. Lecture Notes in Computer Science, 4172, 453-456. Disponible en: http://www. springerlink.com/content/5356711834963732/fulltext.pdf [Consulta: 10/02/2011].

Agenjo Bullón, X., y Hernández Carrascal, F. (2010). La biblioteca virtual: función y planteamiento. Disponible en: http://hdl.handle.net/10760/14352 [Consulta: 17/07/2011].

Anglo American Cataloging Rules (2002-2005). Ottawa: Canadian Library Association.

Austlit (2011). Austilit data models. Disponible en: http://www.austlit.edu.au/about/metadata [Consulta: 10/02/2011].

Bianchini, C., y Guerrini, M. (2009). Quis custodiet ipsos custodies? Observaciones sobre la relación entre FRBR, ICP, ISBD y RDA. Anales de Documentación, 12, 321-341. Disponible en: http://revistas.um.es/analesdoc/article/view/70401/67871 [Consulta: 10/02/2011].

Carlyle, A. (2006). Understanding FRBR as a conceptual model: FRBR and the bibliographic universe. Library Resources \& Technology Services, 50 (4), 264-273.

DCMI/RDA Task Group Workshop (2011). A session at the International Conference on Dublin Core and Metadata Applications. Disponible en: http://lanyrd.com/2011/ dc2011/sfxzw/ [Consulta: 18/7/2011]

Delsey, T. (1997). Modeling the logic of AACR. Paper presented at the International Conference on the Principles and Future Development of AACR, Toronto, Canada, October, 23-25. Disponible en: http://epe.lac-bac.gc.ca/100/200/300/jsc_aacr/modeling/r-bibun. pdf [Consulta: 10/2/2011]. 
Delsey, T. (1998). The logical structure of the Anglo American Cataloging Rules pt 1. Disponible en: http://www.collectioncanada.ca/jsc/docs/aacr.pdf [Consulta: 10/02/2011].

Delsey, T. (1999). The logical structure of the Anglo American Cataloging Rules Pt2. Disponible en: http://www.collectionscanada.ca/jsc/docs/aacr2.pdf [Consulta: 10/02/ 2011].

Delsey, T. (2009). RDA database implementation scenarios. Disponible en: http://www. rda-jsc.org/docs/5editor2rev.pdf [Consulta: 10/02/2011].

Dunsire, G. (2007a). Distinguising content from carrier: The RDA/ONIX framework for resource categorization. D-Lib Magazine, vol. 13 (1/2).

Dunsire, G. (2007b). RDA y sistemas bibliotecarios. BiD Textos universitaris de biblioteconomía i documentació, 19. Disponible en: http://www.ub.edu/bid/19dunsir.htm [Consulta: 10/02/2011].

Estivill Rius, A. (2009). Estado actual de la normativa de catalogación. primera parte: El escenario internacional. BiD Textos Universitaris De Biblioteconomia i Documentació, 22 Disponible en: http://www.ub.edu/bid/22/estivill2.htm [Consulta: 10/02/2011].

Estivill Rius, A. (2011). Nueva normativa de catalogación: pasos hacia un futuro prometedor pero incierto. Anuario ThinkEPI, 162-167.

Gorman, M. (2006). Cataloguing and the third way: An essay on bibliographical control in the digital age. Journal of Library \& Information Science, vol. 32 (1), 5-10.

Heaney, M. (2000). An interview with Tom Delsey. Cataloging and Classification Quarterly, vol. 28 (3), 3-18.

Hernández, A. M. (2010). RDA in BNE. Disponible en: http://www.bne.es/opencms/es/ Servicios/NormasEstandares/DocumentosProfesionales/Docs/RDAenBNE.pdf [Consulta: 10/02/ 2011].

Heath, T., y Bizer, C. (2011). Linked Data: Evolving the Web into a Global Data Space. London: Morgan \& Laypool publishers, 137 p.

Hider, P. (2009). A comparison between RDA taxonomies and user categorizations of content and carrier. Cataloguing and Classification Quarterly, 47 (6), 544-560.

Hillmann. D.; Coyle, K.; Phipps, J., y Dunsire, G. (2010). RDA vocabularies: process, outcome, use. D-Lib Magazine, 16 (2).

IFLA (2007). International standard bibliographic description (ISBD). München: K.G. Saur.

IFLA (2009a). Declaración de Principios Internacionales de Catalogación. Disponible en: http://www.ifla.org/files/cataloguing/icp/icp_2009-es.pdf [Consulta: 10/02/2011].

IFLA (2009b). Functional Requirements for Authority Data: A conceptual model. München: K. G. Saur.

IFLA (2010). Functional Requirements for Subject Authority Data: A conceptual model. Disponible en: http://www.ifla.org/files/classification-and-indexing/functional-requirements-for-subject-authority-data/frsad-model.pdf [Consulta: 18/07/2011].

IFLA (1998). Study Group on the Functional Requirements for Bibliographic Records. Functional Requirements for Bibliographic Records: Final report. München: K.G. Saur.

Joint Steering Committee for development of RDA. (1997). International Conference on the Principles and Future Development of AACR, Toronto, Canada, October 23-25. Disponible en: http://www.rda-jsc.org/intlconf1.html [Consulta: 10/02/2011]. 
Kent State University. School of Library and Information Science (2011). FRBR based systems to effectively support user tasks and facilitate information seeking. Disponible en: http://frbr.slis.kent.edu [Consulta: 10/02/2011].

Le Boeuf, P. (2002). FRBR and further. Cataloging and Classification Quarterly, 32 (4), 15-52.

Library of Congress (2011). Bibliographic framework transition initiative. Disponible en: http://www.loc.gov/marc/transition/ [Consulta: 18/7/2011].

Library of Congress. (2009a). Formato MARC21 conciso para datos bibliográficos. Disponible en: http://www.loc.gov/marc/bibliographic/ecbdspa.html [Consulta: 10/02/2011].

Library of Congress (2009b). FRBR display tool. Disponible en: http://www.loc.gov/marc/ marc-functional-analysis/tool.html [Consulta: 10/02/2011].

Library of Congress (2009c). Propose methodology for US. national libraries RDA test.

Disponible en: http://www.loc.gov/bibliographic-future/rda/testing.html [Consulta: 10/02/2011].

Madison, O. M. A. (2005). The origins of the IFLA study on Functional Requirements for Bibliographic Records. Cataloguing and Classification Quarterly, 39 (4/5), 15-37.

National Library of Australia (2010). Resource description and access (RDA) in Australia. Disponible en: http://www.nla.gov.au/lis/stndrds/grps/acoc/rda.html [Consulta: $15 / 02 / 2011]$

OCLC (2009a). FictionFinder: A FRBR based prototype for fiction in WorldCat. Disponible en: http://www.oclc.org/research/activities/fictionfinder/default.htm [Consulta: 10/02/2011].

OCLC (2009b). Online catalogs: What user and librarians want. Disponible en: http:// www.oclc.org/reports/onlinecatalogs/default.htm [Consulta: 10/02/ 2011].

OCLC. (2010). OCLC policy statement on RDA cataloging in WorldCat for the US testing period. Disponible en: http://www.oclc.org/us/en/rda/policy.htm [Consulta: 10/02/2011].

Oliver, C. (2010). Introducing RDA: A guide to the basics. Chicago: American Library Association.

Open Metadata Registry (2011). The RDA vocabularies. Disponible en: http://metadataregistry.org/rdabrowse.htm [Consulta: 18/07/2011].

Ortiz- Repiso, V. (2005). La catalogación en un entorno híbrido: átomos y bits. En: Orera Orera, L. (Ed.), La biblioteca universitaria (pp. 259-286). Madrid: Sintesis.

Ortiz- Repiso, V.; Moscoso, P. (1999). Web based OPACs: Between tradition and innovation. Information Technology and Libraries, vol. 18 (2), 68-77.

Peset, F.; Ferrer-Sapema, A., y Subirats-Coll, I. (2011). Open data y linked data: su impacto en el área de bibliotecas y documentación. El Profesional de la información, vol. 20 (2), 165-173.

Picco, G., P. A. (2007). RDA: El alcance internacional del nuevo código de catalogación. Transinformação, 19 (3), 219-226.

RDA in Europe: Making it happen. summary of presentations by european countries on plans moving to RDA. (2010). Disponible en: http://www.slainte.org.uk/eurig/docs/ RDA2010/OtherEURIG2010.pdf [Consulta: 15/02/ 2011].

RDA toolkit. (2010). Disponible en: http://www.rdatoolkit.org/ [Consulta: 10/02/2011].

$R D A$, resource description and access (2010). American Library Association, Canadian Library Association, CILIP. 
Salamanca Chiverto, D. (2008). La categorización de contenidos y medios en la descripción bibliográfica: la designación general de material (DGM) y su pasado, presente y futuro en la era digital. Revista Española de Documentación Científica, vol. 31 (4), 527-551.

Svenonius, E. (2000). The intellectual foundation of information organization. Cambridge: MIT Press.

Taylor, A. (2007). An introduction to Functional Requirements for Bibliographic Records (FRBR). En: Taylor, A. (ed.), Understanding FRBR: What is and how it will affect our retrieval tools. Westport, CT.: Libraries Unlimited.

U.S. RDA Test Coordinating Committee (2011). Report and Recommendations. Disponible en http://www.loc.gov/bibliographic-future/rda/rdatesting-finalreport-20june2011.pdf [Consulta: 18/07/2011].

Vellucci, S. (1997). Bibliographic relationships. Paper presented at the International Conference on the Principles and Future Development of AACR, Toronto, Canada, October 23-25. Disponible en http://epe.lac-bac.gc.ca/100/200/300/jsc_aacr/bib_rel/r-bibrel.pdf [Consulta: 10/02/2011].

VTLS (2008). What is virtua? Disponible en http://www.vtls.com/products/virtua [Consulta: 10/02/2011].

W3C Library Linked Data Incubator Group (2010). Incubator Activity. Disponible en: http:// www.w3.org/2005/Incubator/lld/ [Consulta: 18/07/2011].

W3C Library Linked Data Incubator Group (2005). Library Standards. Disponible en: http:// www.w3.org/2005/Incubator/lld/wiki/images/1/12/LayeredModelV3.pdf [Consulta: 18/07/2011].

Zhang, Y., y Salaba, A. (2009). Implementing FRBR in libraries: Key issues and future directions. New York: Neal Schuman. 